\title{
Automated Nomenclature of Bronchial Branches Extracted from CT Images and Its Application to Biopsy Path Planning in Virtual Bronchoscopy ${ }^{\star}$
}

Kensaku Mori ${ }^{1}$, Sinya Ema ${ }^{1}$, Takayuki Kitasaka ${ }^{1}$, Yoshito Mekada ${ }^{2}$, Ichiro Ide $^{1}$, Hiroshi Murase ${ }^{1}$, Yasuhito Suenaga ${ }^{1}$, Hirotsugu Takabatake ${ }^{3}$, Masaki Mori ${ }^{4}$, and Hiroshi Natori ${ }^{5}$

1 Grad. School of Information Science, Nagoya University, Nagoya 464-8603, Japan \{kensaku, kitasaka, suenaga, ide, murase\}@is.nagoya-u.ac.jp

2 School of Life System Science and Technology, Chukyo University, Toyota 470-0393, Japan 3 Minami Sanjo Hospital, Sapporo 060-0033, Japan

4 Sapporo Kosei General Hospital, Sapporo 064-0063, Japan

5 School of Medicine, Sapporo Medical Univerisity, Sapporo 060-8543, Japan

\begin{abstract}
We propose a novel anatomical labeling algorithm for bronchial branches extracted from CT images. This method utilizes multiple branching models for anatomical labeling. In the actual labeling process, the method selects the best candidate models at each branching point. Also a special labeling procedure is proposed for the right upper lobe. As an application of the automated nomenclature of bronchial branches, we utilized anatomical labeling results for assisting biopsy planning. When a user inputs a target point around suspicious regions on the display of a virtual bronchoscopy (VB) system, the path to the desired position is displayed as a sequence of anatomical names of branches. We applied the proposed method to 25 cases of CT images. The labeling accuracy was about $90 \%$. Also the paths to desired positions were generated by using anatomical names in VB.
\end{abstract}

\section{Introduction}

Recent progress in 3-D medical imaging devices has enabled us to measure very precise volumetric data of the human body. Since these imaging devices output a lot of images, development of CAD systems is strongly expected to reduce medical doctors' load and to enable more accurate diagnosis. The bronchus is a very important organ in diagnosis of the lung. In the lung area, it is possible

\footnotetext{
* Parts of this research were supported by Grant-In-Aid for Scientific Research from the Ministry of Education, the 21st-Century COE program, a Grant-In-Aid for Scientific Research from the Ministry of Education, Culture, Sports, Science, and Technology, Japan Society for Promotion of Science, and a Grant-In-Aid for Cancer Research from the Ministry of Health and Welfare.
} 
to define dominant bronchial branches in each segmental lobe or subsegmental area. Bronchial branches connected to lesions are investigated when diagnosing diseases of the lung. Anatomical names of bronchial branches are systematically assigned based on branching patterns. When a medical doctor reviews chest CT images, bronchial branches connected to lesions are identified and their anatomical names are reported. In bronchoscopic examinations, such as transbronchial lung biopsy (TBLB), medical doctors identify appropriate paths for biopsy using the anatomical names on the paths. Automated nomenclature is one of the indispensable functions required in the CAD systems for the lung.

There are only a few reports on automated anatomical labeling of bronchial branches extracted from CT images 1/23. Mori et al. proposed an automated anatomical labeling method where they prepared a branching model containing anatomical names before the labeling process[1]. This model consists of a set of information of each branch, such as running direction, anatomical name, parent branch name, child branch name. Graph representations of the bronchial branches were obtained by applying a thinning method to the bronchus regions. Automated nomenclature (anatomical labeling) process was sequentially performed from the center to the peripheral side by selecting the best fit branch from the model. However, this model is unable to handle variations in branching patterns. Kitaoka et al. proposed an automated labeling method using a graph matching algorithm 2 . It is still difficult to deal with branching pattern variations. The report from Ema et al. proposed a labeling method using multiple models of bronchial branches [3]. They divided the bronchus into four parts and prepared multiple models for each part. For each part, the best model was selected by calculating the evaluation values of fitting and actual labeling was then performed. However, since the model was selected by averaging the differences of the running directions between the models To solve these problems, we propose a new anatomical labeling algorithm that selects the best candidate models at each branching point in Section 2, Also a special labeling procedure is proposed from the right upper lobe. As an application of the automated nomenclature of bronchial branches, we utilize anatomical labeling results for assisting TBLB path planning in Section 4. When a user inputs a target point around suspicious regions on the display of a virtual bronchoscopy (VB) system [4, the path to the desired position is displayed as a sequence of anatomical names of branches. This function will greatly help a bronchoscopist. Experimental results and discussion are described in Sections 5 and 6 .

\section{Bronchial Branch Model}

The bronchial model is a graph representation that shows the branching patterns of the bronchial branches in each lobe in the lung (RU: right upper lobe, RL: right middle and lower lobes, LU: left upper lobe, and LL: left lower lobe) (Fig. 1). The $q$-th model, $M_{q}^{p}$, for the lobe $p$ is a set of attributions of branches $v_{i}$. The $i$-th branch has the following attribute information, $E_{i}$ : branch number $i$, anatomical name $N_{i}$, pointer to parent branch $t_{i}$, running direction $\mathbf{d}_{i}$, pointer 


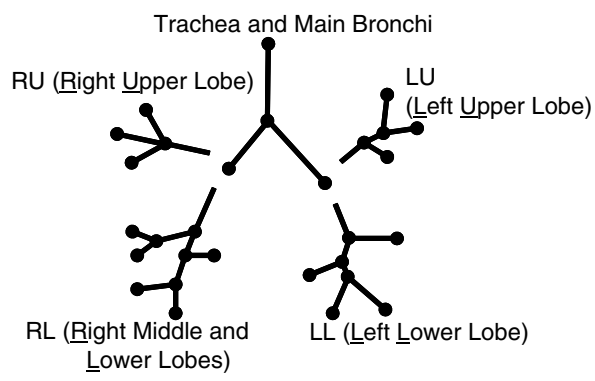

Fig. 1. Division of bronchial trees. A bronchial tree is divided into four lobes: RU, RL, LU, and LL.

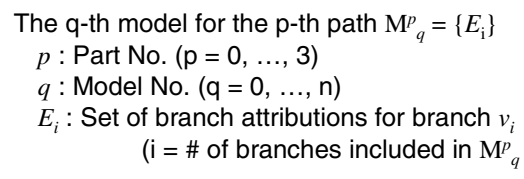

Attribution $E_{i}=\left\{i, N_{i}, N_{i}^{p}, d_{i}, c_{i}\right\}$

$i$ : Branch No. $(=I)$

$N_{\mathrm{i}}$ : Anatomical name

$t_{i}$ : Parent branch No.

$d_{i}$ : Running direction

$t_{i}$ : Child branch No. $\left(\mathrm{j}=1, \ldots, \mathrm{c}_{\mathrm{i}}\right)$

$u_{i}$ : Number of child branches

$\mathrm{s}_{\mathrm{i}}$ : Start position of branch

$\mathrm{e}_{\mathrm{i}}$ : End position of branch

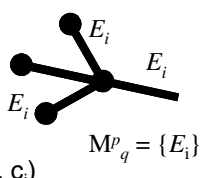

Fig. 2. Branch model structure

to child branches $u_{i}^{j}\left(j=1, \ldots, c_{i}\right)$, number of child branches $c_{i}$, start point $s_{i}$, and end point $e_{i}$ (Fig. 2). These models are prepared for each lobe $(\operatorname{RU}(p=0)$, $\mathrm{RL}(p=1)$, LU $(p=2)$, and LL $(p=3))$ by using hand-labeled bronchial branches extracted from multiple cases of CT images.

\section{Automated Anatomical Labeling}

The actual processing procedure consists of four parts: (a) extraction of bronchial regions and bronchial trees from CT image, (b) labeling of trachea and main bronchi, (c) labeling of the RU, and (d) labeling of remaining lobes (RL, LU, and LL). Bronchus regions and tree structres are extracted by using tree structure tracing algorithm presented in [5].

\subsection{Labeling for RU}

Temporal Labeling. Let $B$ be the child branch of the right upper lobe bronchus (a child branch of the right main bronchus) and a model in the RU $M_{q}^{0}$ is associated with $B$. A labeling process is sequentially performed from $B$ to the peripheral branches by using model $M_{q}^{0}$ prepared for the RU and is saved as the temporal labeling results. Let $B^{\prime}$ be a branch in model $M_{q}^{0}$ that has the same anatomical name as $B$. Model $M_{q}^{0}$ is then rotated around the start point, $B^{\prime}$, so that the running direction of $B^{\prime}$ corresponds to that of $B$. For all possible combinations of one-to-one correspondence between the child branches of $B$ and $B^{\prime}$, the sum of the inner products of running directions of the corresponding pairs is calculated. The combination that produces the minimum sum is considered as the best combination and then copy the anatomical name of $B^{\prime}$ to $B$ and process child branches of $B$ using a breadth-first search. Finally, the labeling results are obtained whose number is equal to the number of the models prepared for RU. 
Model Selection. Using temporal labeling results, inappropriate models are removed from candidate models for the RU. First, three vectors are calculated that start from $B$ and direct to the ends of the branches labeled as Rt. $\mathrm{B}^{1}, \mathrm{Rt}_{\mathrm{B}} \mathrm{B}^{2}$, and Rt.B $B^{3}$ using model $M_{q}^{0}$. These vectors are denoted as $\mathbf{U}_{1}, \mathbf{U}_{2}$, and $\mathbf{U}_{3}$. Also vectors are calculated on model $M_{q}^{0}$ as $\mathbf{V}_{1}, \mathbf{V}_{2}$, and $\mathbf{V}_{3}$ in the same way. If any of the inner products $\mathbf{V}_{1} \cdot \mathbf{U}_{1}, \mathbf{V}_{2} \cdot \mathbf{U}_{2}$, or $\mathbf{V}_{3} \cdot \mathbf{U}_{3}$ is smaller than a given threshold value, the model $M_{q}^{0}$ is removed from the RU model candidates. This process is iterated for all models prepared for the RU.

Final Labeling. The labeling process is performed by using the remaining models. For each model, the model is deformed so that the model fits to the input tree structure and an average deformation angle is calculated. The final labeling is performed using the model whose average deformation angle is the smallest among the models. Anatomical names of the deformed model are assigned to the branches of the input tree structure $[3]$.

\subsection{Labeling for Remaining Parts}

For each child branch of the main bronchi (except for the upper lobe bronchus, i.e. $p=1, \ldots, 3$ ), the following steps are performed (Fig. 3).

1. An anatomical name is assigned to a child branch of a main bronchus (right or left main bronchus) and let it be $O$. The branch $O$ and all candidate models associated with $O$ are stored (for example, if $O$ is left upper lobe bronchus, we associate all models of LU with $O$.) into a FIFO (first in first out) queue.

2. Branch $O$ and a set of models, $\mathbf{M}^{O}$, associated with $O$ are retrieved from the queue. If the queue is empty, the process is terminated.

3. If $O$ does not have a child branch, return to Step 2. Otherwise, branches of $O_{i}^{\prime}$ are searched for whose anatomical names equal $O$ 's anatomical name in each model $M_{i}^{p}$ contained in model set $\mathbf{M}^{O}$. If the number of child branches of $O$ and $O_{i}^{\prime}$ differs, the model $M_{i}^{p}$ is removed from model set $\mathbf{M}^{O}$. The removed model is not used for anatomical labeling of the descendant branches of $O_{i}^{\prime}$.

4. Similarity is calculated between the descendent branches of $O$ and each model $M_{i}^{p}$ of model set $\mathbf{M}^{O}$. First, $M_{i}^{p}$ is rotated around the start point of $O_{i}^{\prime}$ so that the running direction of $O_{i}^{\prime}$ equal the running direction of $O$. For all of possible combinations of one-to-one correspondence between child branches of $O$ and $O_{i}^{\prime}$, the sum of the inner products of the running directions of corresponding pairs is calculated. This sum of the inner products is considered similar between model $M_{i}^{p}$ and the input tree structure.

5. From model set $\mathbf{M}^{O}$, the model, $M_{s}^{p}$, that has the smallest sum is selected and assigned the anatomical names of the child branches $O_{s}^{\prime}$ to the child branch of $O$.

6. The child branch names of $O_{i}^{\prime}$ and $O_{s}^{\prime}$ are compared for each model $M_{i}^{p}$ of model set $\mathbf{M}^{O}$. If there is no branch, whose anatomical name is equal to that of $O_{s}^{\prime}$, in the child branches of $O_{i}^{\prime}$, model $M_{i}^{p}$ is removed from $\mathbf{M}^{O}$.

7. The child branches of $\mathrm{O}$ and the model set $\mathbf{M}^{O}$ are stored into the queue.

8. Return to Step 2 


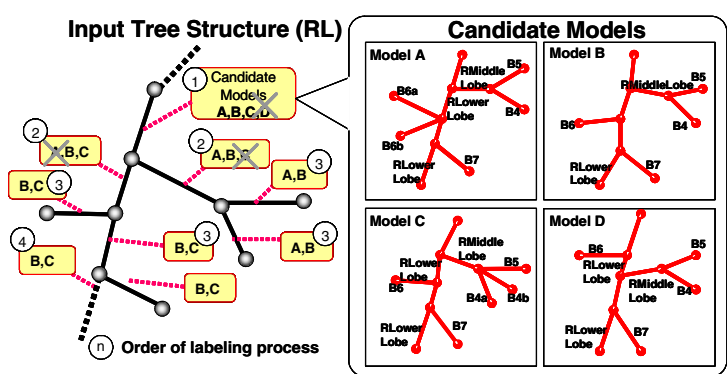

Fig. 3. Illustration of model selection

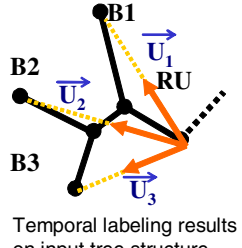
on input tree structure

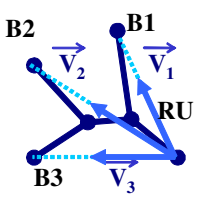

Model $\mathrm{M}_{0}$

Fig. 4. Illustration of vectors $\mathbf{U}_{1}$, $\mathbf{U}_{2}, \mathbf{U}_{3}, \mathbf{V}_{1}, \mathbf{V}_{2}$, and $\mathbf{V}_{3}$

\section{Path Generation for Biopsy Planning}

We integrated the proposed labeling system into the VB system for assisting path planning for TBLB. A physician inputs a desired location on MPR (multi planner reconstruction) images or volume rendering images as end point $\mathbf{p}_{e}$. The start point is set as the start point of the trachea and a branch that is the closest to point $\mathbf{p}_{e}$ is located. In the tree structure of the bronchial branches extracted from CT images, each branch has the information of their path. The path is represented as a set of voxels. By finding the closest point to the specified point, it is possible to select the closest branch. Once the anatomical name of the selected branch is obtained, traverse up the tree structure up to the trachea. Anatomical names of branches existing on the traversed path are displayed on the VB views. Automated fly-through to the desired position is also possible with displaying the anatomical names of the branches on the path.

\section{Experiments and Results}

We applied the proposed method to bronchial branches extracted from twentyfive cases of chest CT images. For each case, a medical doctor assigned anatomical names to all of the branches. Bronchial branch models were constructed from

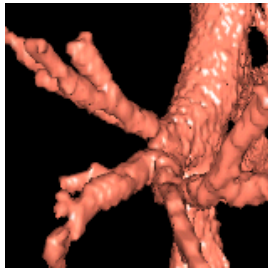

(a) Bronchus regions

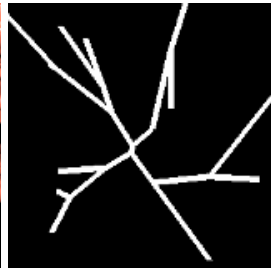

(b) Input tree structure

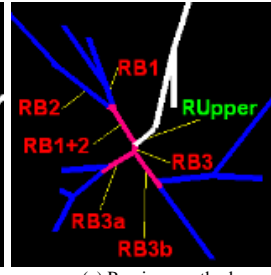

(c) Previous method

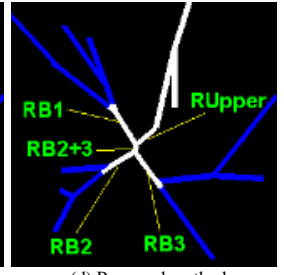

(d) Proposed method

Fig. 5. An example of partial view of bronchus region extracted from CT images (a), its tree structure (b), labeling results using previous (c) and proposed methods (d). Green characters mean correct labeling and red characters mean wrong labeling. 

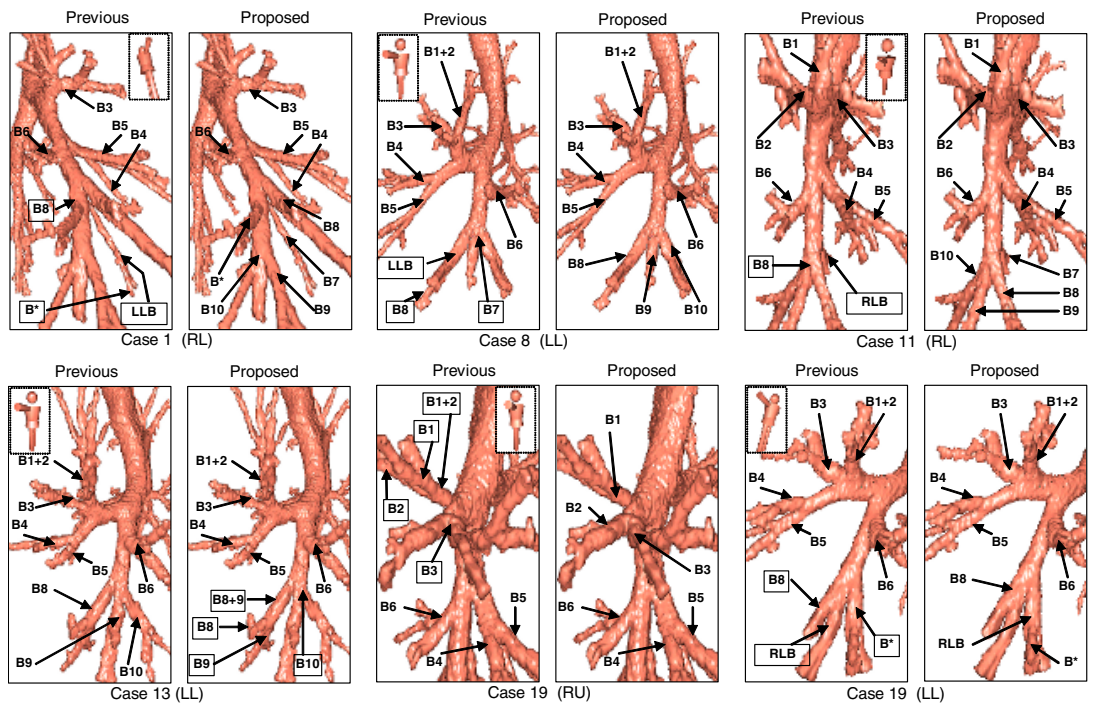

Fig. 6. Labeling results using proposed and previous 3] methods. Anatomical names surrounded by boxes show incorrect labeling.

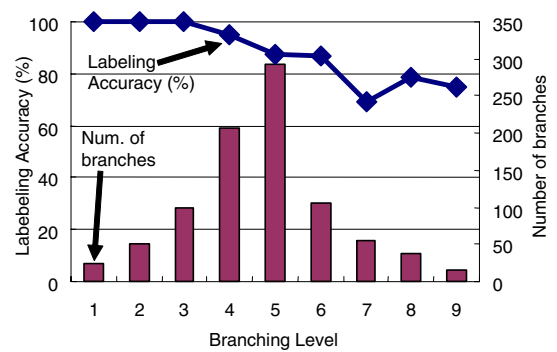

Fig. 7. Number of branches and labeling accuracy at each branching level

24 cases excluding the labeling target case (leave-out-method). We generated six models for the RU, eight models for RL, five models for LU, and six models for LL. We measured labeling accuracy of the proposed and previous methods. Image acquisition parameters of the CT images were: $512 \times 512$ pixels, $141-401$ slices, $0.625-0.723 \mathrm{~mm}$ in pixel spacing, $1.0-2.5 \mathrm{~mm}$ in slice spacing, and $1.25-2.5 \mathrm{~mm}$ of X-ray collimation. All labeling results were evaluated by a medical doctor. Figure 5 shows an example of a partial view of the bronchus region extracted from CT images, its tree structure, and the labeling results using the proposed and previous methods. Table 1 lists the labeling accuracy at each part up to the segmental regions. We successfully assigned correct anatomical names to 799 out of the 888 branches using the proposed method, while 754 branches were assigned using the previous method. The proposed method was 

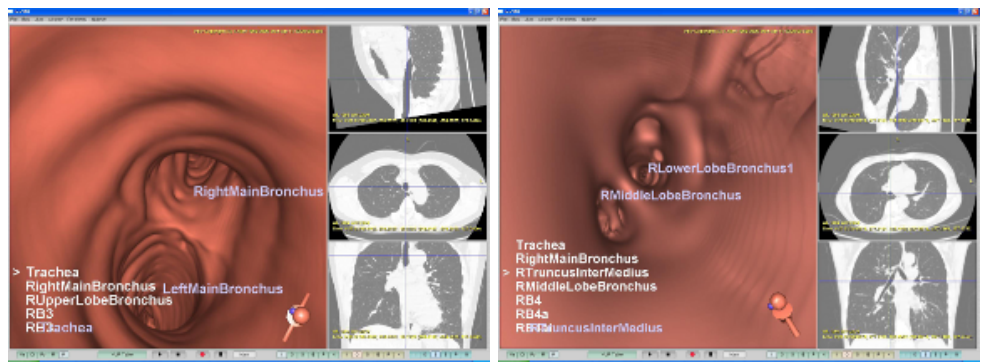

Fig. 8. Path generation for TBLB planning on VB. Anatomical names on the path are displayed on left side of VB view.

able to perform nomenclature on $90 \%$ of the branches extracted from CT images. Examples of anatomical labeling are shown in Fig. 6. Also we measured labeling accuracy at each branching level. Figure 7 shows the number of branches and the correct labeling rate.

We integrated the proposed labeling system into the VB system and tested automated path generation for TBLB path planning. Examples are presented in Fig. 8. In this figure, a user specifies suspicious shadows on slice images by a mouse click. The system automatically generated paths to the specified points and also displayed the paths by using anatomical names of the bronchial branches. These anatomical names are displayed on the left side of a VB view.

\section{Discussion}

From the results listed in Table [1, it is clear that the proposed method significantly improved labeling accuracy in comparison with the previous method. This is because the proposed method selected the appropriate models as branching level progressed. Also, the special model selection procedure for the RU contributed to increase labeling accuracy. Incorrect labeling was prevented by checking dominant regions of the Rt. $\mathrm{B}^{1}$, Rt.B $\mathrm{B}^{2}$, and $\mathrm{Rt} . \mathrm{B}^{3}$ branches. In the labeling result of Case 11, the bronchus bifurcates in the order of Rt.B ${ }^{6}$, Rt. $\mathrm{B}^{7}$, Rt.B ${ }^{8}$, Rt.B ${ }^{9}$, Rt.B ${ }^{10}$. The previous method selected the model having Rt.B* branch and assigned the wrong names after $R t . B^{7}$. The reason for the model mis-selection was the branching pattern and the running directions of branches after Rt. $\mathrm{B}^{6}$ were very close to the input tree structure in the mis-selected model. The proposed method successfully assigned correct names to these branches because the method selected the best models branch by branch and removed inappropriate models from the model set. The special model selection technique worked well in Case 19, as shown in Fig. 6. In this case, the previous method selected the wrong model that assigned the name Rt.B ${ }^{(1+2)}$ to branch Rt. $\mathrm{B}^{1}$. However, in the proposed method, this model was removed by comparing the running directions of Rt.B ${ }^{1}, R t . B^{2}$, and Rt. $B^{3}$ of the model and those of the labeled tree. 
Table 1. Labeling accuracy in segmental level

\begin{tabular}{c|c|c|c}
\hline Part & Number of branches & Previous method [3] & Proposed method \\
\hline Trachea and main bronchi & 75 & $75(100 \%)$ & $75(100 \%)$ \\
RU & 124 & $106(82.5 \%)$ & $110(88.7 \%)$ \\
RL & 322 & $268(83.2 \%)$ & $291(90.4 \%)$ \\
LU & 183 & $159(86.9 \%)$ & $165(90.2 \%)$ \\
LL & 184 & $146(79.3 \%)$ & $158(85.9 \%)$ \\
\hline Total & 888 & $754(84.9 \%)$ & $799(90.0 \%)$ \\
\hline
\end{tabular}

The path for TBLB was automatically generated and described using a set of anatomical names in the proposed method. Also, a user was able to perform fly-through or automated fly-thorough checking anatomical names. Since accessibility to suspicious regions is discussed by using anatomical names of bronchial branches connected to it, it is very useful for medical doctors to see paths by their anatomical names. The automated anatomical labeling system proposed in this paper enabled us to implement function that makes VB systems intelligent.

This paper presented a novel anatomical labeling algorithm for bronchial branches extracted from CT images using multiple branching pattern models. The method presented here is another step towards createing truly intelligent computer aided diagnosis syetm. Annotations displayed on CT images will much assist a medical doctor to read images. Future work includes testing more cases, and improving model selection and removal.

\section{References}

1. K. Mori, J. Hasegawa, Y. Suenaga et al.: Automated Anatomical Labeling of the Bronchial Branch and Its Application to the Virtual Bronchoscopy System. IEEE Transactions on Medical Imaging, Vol 19, 2000.

2. H. Kitaoka, Y. Park, J. Tschirren et al.: Automated Nomenclature Labeling of the Bronchial Tree in 3D-CT Lung Images. T.Dohi and R.Kikinis (Eds.) MICCAI2002, LNCS2489, pp.1-11, 2002.

3. S. Ema, K. Mori, T. Kitasaka, et al.: An Improved method for automated anatomical labeling of bronchial branches extracted from 3-D CT images, In H.U. Lemke, M.W. Vannier, K. Inamura, A.G. Ferman, K. Doi, J.H.C. Reiber eds., Proceedings of the 18th International Congress and Exhibition on Computer Assisted Radiology and Surgery (CARS) 2004, International Congress Series 1268, p.1358, 2004.

4. P. Rogalla, J. Terwisscha van Scheltinga, and B. Hamm, eds.: Virtual endoscopy and related 3D techniques, Springer, Berlin, 2001.

5. T. Kitasaka, K. Mori, J. Hasegawa, et al: A Method for Extraction of Bronchus Regions from 3D Chest X-ray CT Images by Analyzing Structural Features of the Bronchus, FORMA, 17, pp.321-338, 2002. 